\title{
Refining the Role of Simultaneous Liver Kidney Transplantation
}

\author{
Sabiha M. Hussain and Kalathil K. Sureshkumar* \\ Division of Nephrology and Hypertension, Department of Medicine, Allegheny General Hospital, \\ Allegheny Health Network, Pittsburgh, PA, USA
}

\begin{abstract}
Adoption of the model for end-stage liver disease score by Organ Procurement and Transplant Network (OPTN) deceased donor liver allocation policy in 2002 has led to an increase in the number of simultaneous liver kidney (SLK) transplantation. Since kidney function recovery following liver transplantation is difficult to predict, allocation of the kidney for SLK transplantation thus far has not been based on much rationale and evidence. Lack of OPTN policy towards SLK organ allocation has resulted in great variations among transplant centers regarding SLK transplantation. Increasing use of kidneys towards SLK transplantation diverts deceased donor kidneys away from candidates awaiting kidney-alone transplantation. Recently OPTN/United Network of Organ Sharing has implemented medical eligibility criteria for adult SLK transplantation which also includes a concept of safety net. Implementation of the new policy is a move in a positive direction, providing consistency in our practice and evidence-based guidelines in selecting candidates for SLK transplantation. This policy needs to be monitored prospectively and modified based on new data that will emerge over time. This review outlines the literature on SLK transplantation and efforts towards developing rational policy on SLK organ allocation.
\end{abstract}

Citation of this article: Hussain SM, Sureshkumar KK. Refining the role of simultaneous liver kidney transplantation. J Clin Transl Hepatol 2018;6(3):289-295. doi: 10.14218/JCTH.2017.00065.

\section{Introduction}

Renal dysfunction contributes to the morbidity and mortality in liver transplant recipients. Acute kidney injury (AKI) can develop in up to $23 \%$ of patients with cirrhosis, while chronic kidney disease (CKD) is present in $1 \%$ of such patients. ${ }^{1,2}$ Since introduction of the model for end-stage liver disease

Keywords: Liver kidney transplantation; Graft survival; MELD score; Creatinine; GFR.

Abbreviations: AKI, acute kidney injury; CKD, chronic kidney disease; CKD-EPI, Chronic Kidney Disease Epidemiology Collaboration; ESRD, end-stage renal disease; GFR, glomerular filtration rate; KDPI, kidney donor profile index; KTA, kidney transplant alone; LTA, liver transplant alone; MDRD, modification of diet in renal disease; MELD, model for end-stage liver disease; OPTN, Organ Procurement and Transplant Network; RRT, renal replacement therapy; SLK, simultaneous liver kidney; UNOS, United Network of Organ Sharing.

Received: 12 October 2017; Revised: 5 February 2018; Accepted: 22 March 2018 *Correspondence to: Kalathil K. Sureshkumar, Division of Nephrology and Hypertension, Department of Medicine, Allegheny General Hospital, 320 East North Avenue, Pittsburgh, PA 15212, USA. Tel: +1-412-359-3319, Fax: +1-412359-4136, E-mail: kalathil.sureshkumar@wpahs.org
(MELD) scoring system for organ allocation in liver transplant candidates in 2002, the incidence of renal dysfunction among liver transplant recipients has been significantly increasing, contributing to an increasing trend in simultaneous liverkidney transplantation (SLK) after 2002. ${ }^{3-5}$

AKI affects $25 \%$ to $50 \%$ of liver transplant recipients, while CKD develops in $30 \%$ to $90 \%$ and risk of end-stage renal disease (ESRD) requiring renal replacement therapy (RRT) is in the range of $2 \%$ to $5 \%$ per year after transplantation. ${ }^{6}$ Native kidney function recovery following liver transplantation alone (LTA) is difficult to predict, and indications for SLK transplantation are not precisely defined. The decision to transplant SLK has largely been center-specific and driven by the fear of persistent renal failure after LTA and its associated poor outcomes. A recent study identified early liver allograft dysfunction, early development of stage 3 AKI following LTA and requirement for RRT at the time of liver transplantation as independent risk factors for the development of ESRD within first year of LTA. ${ }^{7}$

Predictors of renal function recovery with good discrimination following LTA are poorly defined. Patients wait-listed for kidney transplantation after nonrenal organ transplantation have worse outcomes compared to patients waiting for kidney transplant alone (KTA). ${ }^{8}$ Currently, the outcomes of SLK transplants are excluded from Scientific Registry of Transplant Recipients, further encouraging the transplant centers to add kidney to a high-risk liver candidate in order to buffer their program's outcome. ${ }^{4}$ Lack of uniform criteria for allocating kidney to SLK has led to marked variation in the rates of SLK transplantation among transplant centers, ranging from $0 \%$ to $44 \%$ of all liver transplants performed. ${ }^{9}$ With the increasing trend in the number of SLK transplants has come controversy over whether the addition of kidney allograft to liver transplant candidates with renal dysfunction is associated with superior long-term outcomes, or is it an unnecessary use of a limited resource in the era of increasing organ shortage.

\section{Impact of renal function on postliver transplant survival}

Pretransplant renal function was found in earlier studies to be an independent predictor of survival following liver transplantation. ${ }^{3,5}$ This is particularly true among patients with serum creatinine greater than $2 \mathrm{mg} / \mathrm{dL}$ or requiring prolonged RRT prior to undergoing liver transplantation. Moderate and severe renal dysfunction was reported as associated with poor graft and patient survival following LTA in a United Network of Organ Sharing (UNOS) registry analysis. ${ }^{5}$ In a study performed by Gonwa et al., ${ }^{3}$ 5-year patient survival rates following deceased donor LTA were $79.1 \%, 72.2 \%$, and $63.1 \%$ in 
patients with pretransplant serum creatinine levels of $0-0.99$ $\mathrm{mg} / \mathrm{dL}, 1.0-1.99 \mathrm{mg} / \mathrm{dL}$, and $>2.0 \mathrm{mg} / \mathrm{dL}$ respectively. Corresponding survivals were $63.9 \%$ in patients who required RRT at the time of deceased donor LTA and $69.6 \%$ in those who underwent SLK transplantation. Patients requiring RRT preoperatively had better survival with SLK transplantation compared to deceased donor LTA. Because of the impact of renal function on postliver transplant survival, serum creatinine was included as a variable in the MELD scoring system.

\section{MELD, postMELD era and need for SLK policy}

MELD was developed to estimate the mortality in patients waiting for liver transplantation and to allocate organs to the sicker patients who would gain the most benefit from transplantation. The components of MELD score include the international normalized ratio, serum bilirubin and serum creatinine. The Organ Procurement and Transplant Network (OPTN)/ UNOS adopted the MELD scoring system in February 2002 to facilitate liver allograft allocation to wait-listed patients based on medical necessity in compliance with OPTN's "Final Rule", which states that "allocation policies must be based on sound medical judgment and standardized criteria, must seek to achieve the best use of organs and must avoid futile transplants". Since MELD score heavily weighs serum creatinine, it has led to an increased number of patients with severe renal dysfunction and on RRT to undergo liver transplantation.

An unintended consequence of the introduction of MELD scoring for liver allocation in 2002 was a steady increase in SLK transplantation, from 135 in 2000 to 731 in 2016 nationally, as shown in Fig. 1. The question regarding the benefits of SLK over LTA in patients with renal dysfunction then arises. Multiple single-center studies and registry analyses aimed to address the added benefits of the addition of a kidney transplant to liver transplant candidates with renal dysfunction have shown variable results. ${ }^{4,10,11} \mathrm{~A}$ matched-control analysis by Locke et al. ${ }^{10}$ utilizing the UNOS database in 2008 failed to show benefit of SLK transplantation over LTA despite using higher quality allografts for SLK transplantation. This could likely be related to the fact that kidney grafts were allocated to sicker recipients, often with AKI, who probably developed multiorgan disease processes that were too advanced to benefit from either mode of transplantation. A subgroup analysis suggested patient and liver allograft survival benefits for those SLK recipients who were on dialysis for more than 3 months prior to

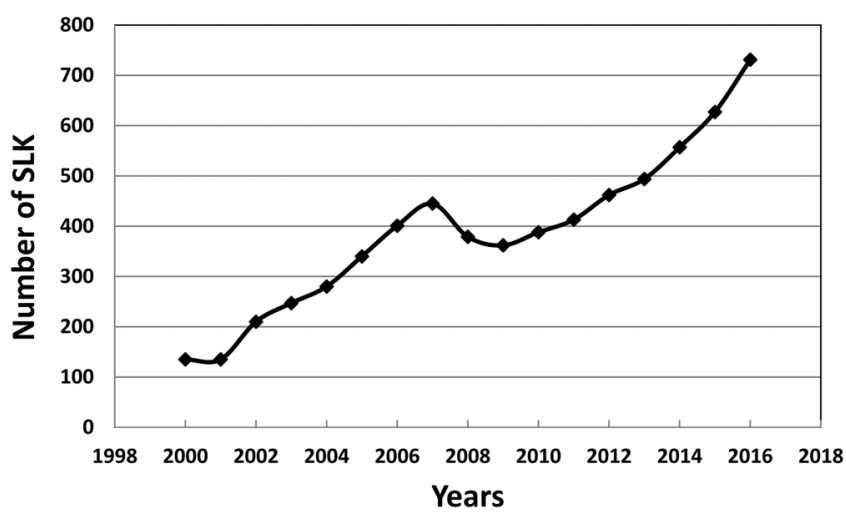

Fig. 1. SLK transplantation by year in the USA. Abbreviation: SLK, simultaneous liver kidney. transplantation as compared to LTA. $^{10}$ Another study by Gonwa et al. ${ }^{3}$ comparing the patient survival between SLK and LTA revealed that patients on RRT at the time of liver transplantation do better with SLK than LTA.

A recent review of the literature and OPTN database analysis regarding recipient survival with or without renal dysfunction after SLK transplantation by Formica et al. ${ }^{4}$ showed that $37 \%$ of SLK recipients received no dialysis prior to transplantation and out of those who received no dialysis, $40 \%$ had creatinine $<2.5 \mathrm{mg} / \mathrm{dL}$ at time of transplantation. The authors also illustrated that liver transplant candidates with renal failure (defined by pretransplant dialysis time of $>2$ months or serum creatinine $>2.5 \mathrm{mg} / \mathrm{dL}$ ) benefited from SLK transplantation compared to LTA. However, the outcomes with SLK were inferior compared to those of LTA in patients without renal dysfunction. A propensity score matched study comparing 1884 SLK recipients with 31,882 LTA recipients transplanted from 2002 to 2009 showed a small survival benefit of 3.7 months at 5 years in patients with pretransplant nondialysisdependent CKD who underwent SLK compared to LTA. ${ }^{12}$ The consensus finding from the registry studies is that SLK is beneficial for those liver transplant candidates with marked renal dysfunction, or for those who have been on prolonged pretransplant dialysis. ${ }^{3,4,11}$

Kidney allograft survival is remarkably inferior in SLK recipients as compared to KTA. ${ }^{4,10,13}$ In fact, the review by Schinzler et al. ${ }^{14}$ showed that any of the kidney allografts that failed following SLK transplantation in the MELD era would have added a graft lifespan of 7.2 years, if transplanted to a candidate on KTA wait-list. OPTN policy prioritizes organ allocation to multiorgan candidates before kidney-alone candidates when the candidate is in the same donor service area as the donor. ${ }^{4} \mathrm{~A}$ paired kidney analysis of multiorgan transplantation by Choudhary et al. ${ }^{13}$ demonstrated inferior survival when a kidney is allocated to an SLK recipient compared to the contralateral mate kidney allocated to KTA. Kidneys used for SLK transplants have lower kidney donor profile index (KDPI) and thus have higher expected longevity. ${ }^{15}$ Among the SLK transplants, $49 \%$ of donor kidneys had a KDPI $<35 \%$, that would likely be offered to pediatric patients on the KTA waitlist. On an average, 250 high-quality donor kidneys per year are being utilized by SLK transplants that otherwise would have been offered to one of the prioritized groups on the KTA wait-list, such as pediatric, young adult or highly sensitized patients.

It is not entirely clear which patients with renal dysfunction would benefit from SLK. Centers err on the side of caution while selecting candidates for SLK, as it is hard to predict if the renal dysfunction prior to liver transplant is reversible or not. There have not been standard medical criteria for the evaluation of patients with renal dysfunction in advanced liver disease requiring liver transplantation.

Is renal dysfunction irreversible in a potential liver transplant candidate?

The decision whether a liver transplant candidate with renal dysfunction should receive SLK versus LTA depends on the ability to predict whether the kidney disease is reversible or not. Renal function is commonly assessed by using a serum creatinine-based formula to calculate glomerular filtration rate (GFR), which is not very reliable in patients with liver disease. Muscle mass, proximal tubular secretion and medications can affect serum creatinine level. For instance, trimethoprim 
increases serum creatinine level by affecting tubular creatinine secretion without altering GFR.

In patients with chronic liver disease, the relatively lower serum creatinine is related to poor muscle mass, decreased hepatic conversion of creatine to creatinine, and increased volume of distribution due to the accumulation of extracellular fluid, all of which can lead to overestimation of GFR when creatinine-based equations are used. In addition, elevated serum bilirubin interacts with creatinine assay, giving falsely low serum creatinine results. The relatively low serum creatinine can mask the drop in GFR in this patient population. This effect is even more pronounced in women, who generally have lower muscle mass to begin with, resulting in over-estimation of GFR.

Different creatinine-based GFR estimation equations are available, such as the Cockcroft-Gault equation, the modification of diet in renal diseases (MDRD) study equation and the Chronic Kidney Disease Epidemiology Collaboration (CKD-EPI) equation. The Cockcroft-Gault equation is based on the assumption that creatinine production decreases with advancing age and is higher in individuals with greater weight but does not take into account obesity with increased body fat. This equation has not been revised for use with creatinine values traceable to standardized reference. The MDRD equation was primarily derived from white subjects with nondiabetic kidney disease and is less accurate in populations with normal or near-normal GFR. The CKD-EPI equation is more accurate than the MDRD equation at higher levels of GFR and in subgroups defined by sex, race, diabetes and transplant status, older age and higher body mass index.

Cystatin C-based equations may be superior to serum creatinine in estimating GFR, as they are not affected by muscle mass, but the cystatin $C$ assay may not be readily available. ${ }^{16}$ Combining both serum creatinine and cystatin $C$ into a single equation can provide more precise estimated GFR compared to equations that use cystatin C or creatinine alone. The best methods for assessment of GFR in patients with cirrhosis depend on the clearance of exogenous markers, such as iothalamate, $51 \mathrm{Cr}$-ethylenediaminetetraacetic acid and inulin, but these measurements are expensive, laborious and not readily available. In addition, the clearances of exogenous markers to estimate GFR have not been thoroughly studied in patients with advanced liver disease and ascites.

It is crucial to differentiate AKI from CKD in liver transplant candidates. CKD defined as GFR $<60 \mathrm{~mL} / \mathrm{min}$ for 3 months or longer can be present in patients with chronic liver disease. Recently, nonalcoholic fatty liver disease has been recognized as a risk factor for CKD. ${ }^{17}$ Many patients with nonalcoholic fatty liver disease also have underlying diabetes mellitus. Patients with hepatitis $\mathrm{C}$ and $\mathrm{B}$ might have coexisting glomerulonephritis. AKI in advanced liver disease is commonly due to three main etiologies: prerenal, hepatorenal syndrome, and acute tubular necrosis. Differentiating between these etiologies has important implications towards management and prognosis. For instance, hepatorenal syndrome is a reversible functional renal injury to the kidneys in the setting of advanced liver disease, which does not generally qualify for the SLK transplant. AKI is also common following liver transplantation and is multifactorial in etiology, ranging from acute tubular necrosis due to hemodynamic instability to drug toxicity.

During the immediate postliver transplant period, about $8 \%$ to $17 \%$ of patients require RRT. ${ }^{16}$ About $68 \%$ recovered renal function, defined as removal from RRT without death or need for renal transplantation after liver transplantation, who were on RRT at the time of transplantation. ${ }^{18}$ The majority of patients who recovered renal function were on RRT for $<30$ days in the pretransplant period. Ojo et al. ${ }^{19}$ utilized registry data on nonrenal transplant recipients and found an $18 \%$ incidence of CKD at 5 years after liver transplantation. Ruebner et al. ${ }^{20}$ looked at the risk of developing ESRD among patients with renal dysfunction at the time of liver transplantation. The highest risk for developing ESRD at 3 years after LTA was $31 \%$ in those with eGFR consistently $<30 \mathrm{~mL} / \mathrm{min}$. At 3 years after LTA, only $6 \%$ of patients who had received short-term dialysis before transplantation were still on dialysis. LTA recipients who required pretransplant RRT for $<30$ days are likely to recover renal function spontaneously postliver transplant, whereas those on RRT for $>90$ days do not. ${ }^{21}$

Previous studies have attempted to generate equations based on several candidate variables in order to predict the development of postliver transplant ESRD with good discrimination and C-statistics ranging from $0.74-0.76 .^{22,23} \mathrm{Com}-$ monly cited predictors of ESRD development following LTA include duration and severity of CKD, level of chronicity on kidney biopsy, recipient age, duration of diabetes and hepatitis $C$ virus status. A renal biopsy is gold standard to assess the chronicity of the kidney disease but carries increased bleeding risk in a cirrhotic patient with coagulopathy. Progression of underlying renal disease can be correlated with the degree of interstitial fibrosis, glomerulosclerosis and arteriolosclerosis. In one study, 59 liver transplant candidates with renal impairment underwent kidney biopsy and SLK was recommended for patients with $>40 \%$ global glomerulosclerosis, $>30 \%$ interstitial fibrosis or requiring dialysis for $>2$ months. Based on these criteria, $70 \%$ of listed patients did not undergo SLK; twenty-three ultimately underwent LTA and ten patients underwent SLK. There were no differences in renal function and survival at 1 year. Biopsy-related complications developed only in two patients. ${ }^{24}$

\section{Principles involved in designing an SLK allocation system}

Attempts have been made by different societies involving transplant surgery, hepatology and nephrology to come up with SLK allocation, as summarized in Table 1 . These policies have sought to address the following areas: i) most accurate and cost-effective way to measure renal function and diagnose kidney disease in patients with cirrhosis; ii) predictors of irreversible kidney function in candidates being evaluated for simultaneous liver kidney transplant; iii) impact of kidney after liver transplantation on the outcomes in patients with persistent renal dysfunction following LTA.

\section{Proposed new policy for SLK allocation}

In order to develop a proposal for policy on SLK allocation, Formica et al. ${ }^{4}$ analyzed the OPTN database to evaluate the characteristics of SLK recipients, outcomes in patients with and without kidney disease after liver transplantation, waitlist survival in those awaiting primary kidney transplantation versus those waiting for kidney after liver transplantation, and kidney graft survival following KTA versus SLK transplantation. The authors concluded that patients with liver transplantation with renal failure defined as pretransplant dialysis duration $>2$ months or serum creatinine $>2.5 \mathrm{mg} / \mathrm{dL}$ benefited from SLK, whereas SLK could be detrimental for those without renal failure. The proposal included the medical eligibility criteria 
Hussain S.M. et al: Simultaneous liver-kidney transplantation

Table 1. Published guidelines and policies towards simultaneous liver and kidney transplantation

\begin{tabular}{|c|c|}
\hline Author, year & Guidelines and policies \\
\hline Davis et al., ${ }^{25} 2007$ & $\begin{array}{l}\text { - } \text { CKD defined as } \mathrm{CrCl} \leq 30 \mathrm{~mL} / \mathrm{min} \text { for }>3 \text { months } \\
\text { - AKI with or without hepatorenal syndrome requiring dialysis for } \geq 6 \text { weeks } \\
\text { - Persistent AKI with biopsy evidence of irreversible damage } \\
\text { - For patients with AKI not on dialysis, SLK is not recommended }\end{array}$ \\
\hline Eason et al., ${ }^{9} 2008$ & $\begin{array}{l}\text { - End-stage renal disease } \\
\text { - CKD with GFR } \leq 30 \mathrm{~mL} / \mathrm{min} \\
\text { AKI with or without hepatorenal syndrome with serum creatinine } \geq 2 \mathrm{mg} / \mathrm{dL} \text { and dialysis requirement } \geq 8 \text { weeks } \\
\text { - CKD with kidney biopsy evidence for }>30 \% \text { glomerulosclerosis or } 30 \% \text { fibrosis } \\
\text { - Other recommended criteria for SLK consideration: comorbidities such as diabetes, hypertension, age }>65 \text { years, } \\
\text { and chronicity of kidney disease based on creatinine, proteinuria and kidney size }\end{array}$ \\
\hline OPTN Policy 3.5.10 2009 & $\begin{array}{l}\text { - } \text { CKD with dialysis need } \\
\text { - } \text { SKD }(\mathrm{GFR} \leq 30 \mathrm{~mL} / \mathrm{min} \text { and proteinuria }>3 \mathrm{~g} / \text { day) } \\
\text { - Sustained AKI with dialysis need for } 6 \text { weeks or longer (dialysis at least twice per week) } \\
\text { - Metabolic disease }\end{array}$ \\
\hline Nadim et al., ${ }^{26} 2012$ & $\begin{array}{l}\text { - Persistent AKI } \geq 4 \text { weeks with one of the following: } \\
\text { - Increase in serum creatinine } \geq 3 \text {-fold from baseline or on dialysis } \\
\text { - GFR } \leq 35 \mathrm{~mL} / \mathrm{min} \text { (MDRD- } 6 \text { ) or } \leq 25 \mathrm{~mL} / \mathrm{min} \text { (iothalamate) } \\
\text { - CKD } \geq 3 \text { months with one of the following: } \\
\text { - estimated GFR } \leq 40 \mathrm{~mL} / \mathrm{min} \text { (MDRD- } 6 \text { ) or } \leq 30 \mathrm{~mL} / \mathrm{min} \text { (iothalamate) } \\
\text { - Proteinuria } \geq 2 \mathrm{~g} / \text { day } \\
\text { - Kidney biopsy showing }>30 \% \text { glomerulosclerosis or }>30 \% \text { interstitial fibrosis }\end{array}$ \\
\hline Formica et al., ${ }^{4} 2016$ & $\begin{array}{l}\text { - CKD: estimated GFR of }<60 \mathrm{~mL} / \mathrm{min} \text { for }>90 \text { days prior to listing and an estimated GFR of }<35 \mathrm{~mL} / \mathrm{min} \\
\text { at the time of listing } \\
\text { - Sustained AKI: a combination of dialysis and estimated GFR }<25 \mathrm{~mL} / \mathrm{min} \text { for } 6 \text { consecutive weeks' duration } \\
\text { - Setabolic disease } \\
\text { - Regional ner kidney after liver transplantation } \\
\text { Reging of kidney for SLK with high MELD score }\end{array}$ \\
\hline
\end{tabular}

Abbreviations: AKI, acute kidney injury; CKD, chronic kidney disease; GFR, glomerular filtration rate; MELD, model of end stage liver disease; MDRD, modification of diet in renal disease.

for SLK transplant and the concept of a safety net for liver recipients who develop ESRD shortly after LTA.

Medical eligibility criteria for SLK transplantation includes patients with CKD, sustained AKI and metabolic disease. CKD was defined as estimated GFR of $<60 \mathrm{~mL} / \mathrm{min}$ for $>90$ days prior to listing and an estimated GFR of $<35 \mathrm{~mL} / \mathrm{min}$ at the time of listing or ESRD on maintenance dialysis. The rationale for suggesting a higher GFR cut-off for SLK listing is based on the data showing a $30 \%$ frequency of ESRD over 3 years in patients who had an GFR $<30 \mathrm{~mL} / \mathrm{min}$ at the time of liver transplantation. ${ }^{20}$ Moreover, the addition of calcineurin inhibitor to the immunosuppressive regimen in patients with low GFR results in a further reduction in GFR, by at least $10 \mathrm{~mL} / \mathrm{min}$, soon after transplantation, bringing the GFR closer to the GFR requirement for KTA listing. ${ }^{27,28}$ Sustained AKI was defined as the requirement for acute dialysis for 6 weeks or longer, an estimated GFR $<25 \mathrm{~mL} / \mathrm{min}$ for 6 weeks or more or a combination of dialysis and estimated GFR $<25 \mathrm{~mL} / \mathrm{min}$ for 6 consecutive weeks duration.

The concept of a safety net is an essential component of the proposed policy. This stems from the evidence that survival of LTA recipients on the kidney wait-list is inferior to those listed for first KTA. However, those prior LTA recipients who developed kidney failure and received a kidney transplant within 3 years of the liver transplant had long-term survival that was similar to KTA recipients. It was proposed that any LTA recipient who is registered for the kidney wait-list between 60 to 365 days postliver transplant with either ESRD or GFR $\leq 20 \mathrm{~mL} / \mathrm{min}$ should be prioritized. It will not be applicable to SLK recipients, unless they meet the definition of primary kidney nonfunction (defined as being on dialysis or estimated GFR $\leq 20 \mathrm{~mL} / \mathrm{min}$ ) 90 days after SLK transplantation. The current UNOS criteria for simultaneous liver kidney transplantation including "safety net" are shown in Table 2. This policy went into effect as of August $10,2017.29$

Another feature added to the policy was inclusion of regional sharing of kidneys in SLK transplant for patients with MELD scores $\geq 35$. Prior to implementation of this SLK policy, patients requiring SLK transplant are likely to have MELD scores $\geq 35$ and are eligible for regional sharing for liver but not for kidney under the new kidney allocation policy. Thus, physicians were forced to make a choice of either waiting for SLK when offered locally or to simply accept liver-alone through regional sharing for patients with high MELD score.

\section{Predicted outcomes from the proposed and recently implemented SLK policy}

The policy proposed for SLK by Formica et al. ${ }^{30}$ is a first step towards optimizing the use of a scarce resource. The intent of the policy is to standardize the allocation of kidney allograft in liver transplant recipients based on medical eligibility. Adoption of new criteria can reduce variability in center-wise practice patterns, better benchmarking of practice and further refinement of SLK allocation recommendations. ${ }^{30}$

The key feature of this policy of requiring a safety net will allow patients who suffer from ESRD soon after liver transplantation timely access to kidney transplantation. It is hoped that this will decrease the physician's rushed and erratic conclusion to add kidney allograft in prospective liver transplant recipients with potentially reversible renal dysfunction and relieve the clinicians of the burden of a wrong decision.

Almost $15-20 \%$ of LTA candidates have an estimated GFR $<30 \mathrm{~mL} / \mathrm{min}$ and another $20-30 \%$ have an estimated GFR $<60 \mathrm{~mL} / \mathrm{min}$ at the time of liver transplant evaluation. ${ }^{6,22}$ The risk of ESRD at 1 year from LTA is minimal, even in 
Hussain S.M. et al: Simultaneous liver-kidney transplantation

Table 2. Current UNOS criteria for simultaneous liver kidney transplantation including "safety net"

Confirmation of diagnosis needed:

Required documentation in patient's medical record and report in UNOS computer system:

CKD, defined as either measured or calculated GFR $\leq 60 \mathrm{~mL} / \mathrm{min}$ for $>90$ consecutive days

\section{Sustained AKI}

Metabolic disorders

"Safety Net": Additional priority will apply to all LTA recipients as well as SLK recipients who experienced immediate and permanent non-function of the transplanted kidney who are on kidney waiting list after becoming dialysis-dependent or having a GFR $\leq 20 \mathrm{~mL} / \mathrm{min}$ between 60 and 365 days following liver transplantation
At least one of the following:

- Maintenance dialysis

- Most recent measured or calculated creatinine clearance or GFR $\leq 30 \mathrm{~mL} / \mathrm{min}$ at the time of registration

- Measured or calculated creatinine clearance or GFR $\leq 30 \mathrm{~mL} / \mathrm{min}$ on a date after registration on kidney wait list

At least one of the following or combination of both of the following for the preceding 6 weeks:

- On dialysis at least once every 7 days

- Measured or calculated creatinine clearance or GFR $\leq 25 \mathrm{~mL} / \mathrm{min}$ at least once every 7 days

- If eligibility is not confirmed once every 7 days for the previous 6 weeks, then the candidate is not eligible to receive liver and a kidney from the same donor

At least one of the following diagnoses:

- Hyperoxaluria

- Atypical HUS due to factor $\mathrm{H}$ or factor I mutation

- Familial nonneuropathic systemic amyloidosis

- Methylmalonic aciduria

- Confirmation at least once every 30 days that the eligibility criteria continue to be met

- Once the program confirms eligibility criteria for three consecutive 30-day periods after the initial qualifying date, the candidate will remain eligible for safety net priority indefinitely

Abbreviations: AKI, acute kidney injury; CKD, chronic kidney disease; GFR, glomerular filtration rate; HUS, hemolytic uremic syndrome; LTA, liver transplantation alone; SLK, simultaneous liver kidney transplantation; UNOS, United Network of Organ Sharing.

Adapted from https://optn.transplant.hrsa.gov/media/1240/05_slk_allocation.pdf

recipients with listing estimated GFR $<35 \mathrm{~mL} / \mathrm{min}$ who were not on RRT at the time of transplantation. ${ }^{31,32}$ Hence, one could argue the medical eligibility criteria of GFR $<35 \mathrm{~mL} / \mathrm{min}$ at the time of listing in the proposed policy as liberal. There is possibility of abuse with these criteria for listing of SLK if there is one time documentation of GFR $<35 \mathrm{~mL} / \mathrm{min}$. There is also concern that this policy might in fact further increase the rate of SLK transplantation. ${ }^{33}$ It is also possible that the new criteria could potentially decrease the number of SLK transplants performed, since 19\% all SLK transplants performed between 2014 and 2015 did not meet current proposed criteria.

Proven factors for CKD, such as the presence of long standing pretransplant diabetes or proteinuria, were not incorporated in the proposed policy. ${ }^{34}$ More controversial and liberal is the AKI definition in the policy. ${ }^{30}$ These patients comprise about $40 \%$ of all SLK transplantations. Less than $10 \%$ of patients with LTA are on dialysis in the first year of transplantation, indicating that many recover native renal function. ${ }^{22}$ Asch et al. ${ }^{30}$ also propose the concept of futility to be added to the policy, where organs are not being offered to patients with poor projected survival. ${ }^{30}$

The proposed policy of SLK is based on the tenet of urgency, which contradicts the new kidney allocation system based on the tenet of utility. SLK transplantation exemplifies the tradeoff between the principle of utility and medical urgency, as shown by a recent Scientific Registry of Transplant Recipients database analysis that compared kidney allograft life span in SLK recipients to donor-matched kidneys transplanted as KTA or simultaneous pancreas kidney transplantation. The adjusted 10-year mean kidney allograft life span was higher in KTA and simultaneous pancreas kidney recipients compared with SLK recipients by 0.99 years in the MELD era and 1.71 years in the preMELD era. In other words, about 1 year of renal allograft life span is traded with SLK transplantation, so that a sicker patient gains access to organs. ${ }^{35}$

The proposed policy for SLK transplantation does not address the current multiorgan transplantation policy, which conflicts with the OPTN's "final rule" based on sound medical judgment to ensure equity and efficacy in organ allocation. The current multiorgan transplant policy provides kidney allocation with another organ, regardless of the degree and duration of renal dysfunction, potential of kidney function recovery and the survival benefit of the addition of kidney. ${ }^{34}$

A recent study by Ekser et al., ${ }^{36}$ described a novel idea of delaying the kidney allograft for $>48 \mathrm{~h}$ in patients undergoing combined liver kidney transplantation. ${ }^{36}$ Liver transplantation is first performed while the kidney allograft is placed on the hypothermic pulsatile machine in order to allow time to stabilize the patient's hemodynamics and normalize coagulopathy before implantation of the kidney allograft, thus reducing the risk for delayed graft function development and optimizing the renal allograft function. This approach was associated with superior graft and patient survival rates during the 4 year follow-up. This could also avoid the wastage of kidney allograft in high-risk liver transplant patients.

Finally, the interesting question as to whether liver is immune-protective to the kidney in the setting of SLK transplantation has been asked. A recent study looked at the incidence of kidney allograft rejection between SLK $(n=68)$ and KTA $(n=136)$ recipients, utilizing protocol renal transplant biopsies. ${ }^{37}$ Pretransplant donor-specific antibodies were present in $20.5 \%$ of the patients in both groups. Among patients with donor-specific antibodies, there was higher incidence of antibody-mediated rejection (46.4\% vs. $7.1 \%$ ) and transplant glomerulopathy $(53.6 \%$ vs. $0 \%$ ) in KTA versus SLK recipients. 
- Reduced kidney function is a predictor of adverse outcomes in liver transplant recipients

- Burden of kidney disease is relatively high in patients with liver disease awaiting transplantation

- Number of SLK transplantation is on the rise since the introduction of the MELD scoring system for liver allocation in 2002

- Indications for SLK transplantation are not precisely defined with center-wide practice variation

- Measuring kidney function with serum creatinine level has significant limitation in patients with liver disease in whom cystatin-C-based equations may be more reliable but not widely available

- Kidneys used in SLK allocation tend to have lower KDPI which would otherwise have been allocated to pediatric patients on the waiting list for kidney alone transplantation

- There is a great need for the standardization of kidney allograft allocation for SLK transplantation in order to balance the benefits of this procedure with the downside of not being able to utilize that kidney for a patient awaiting kidney-alone transplantation

- The newly proposed and recently implemented policy includes medical eligibility criteria for SLK allocation and a concept of "safety net" for those liver recipients who develop ESRD shortly after transplantation along with a recommendation for regional sharing of kidneys for SLK transplantation

- This policy is a step in the right direction and should be modified based on new data that will emerge after its implementation

Abbreviations: ESRD, end-stage renal disease; KDPI, kidney donor profile index ; MELD, model for end-stage liver disease; SLK, simultaneous liver kidney.

Among patients with no donor-specific antibodies, KTA recipients experienced higher incidence of T cell-mediated rejection (30.6\% vs. $7.4 \%$ ) and declining renal allograft function, while SLK recipients has stable GFR. This suggests a protective effect of liver allograft against chronic immunologic injury.

\section{Concluding remarks}

Key points regarding the current status of SLK transplantation are summarized in Table 3. The decision to perform SLK transplant rather than LTA is important because the benefits to the patient with liver disease of receiving a kidney transplant must be balanced with the downside of not being able to use that organ for a patient with ESRD. Inappropriate utilization of this scarce resource may be detrimental to the ever-growing population of patients awaiting kidney transplantation. Furthermore, the kidney grafts that are transplanted into SLK recipients are from higher quality donors, emphasizing more on appropriate allocation of the best deceased donor kidneys. Although the MELD score was designed to reduce wait-list mortality, kidney or liver transplant futility among SLK transplant recipients with the highest level of acuity should not be overlooked. Although not yet readily available in many centers, cystatin C-based equations may allow clinicians to better select the most appropriate candidates for SLK or LTA.

Implementation of the new policy is a move in a positive direction, providing consistency in our practice and evidencebased guidelines in selecting candidates for SLK transplantation. This policy needs to be monitored prospectively and modified based on new data that will emerge over time.

\section{Conflict of interest}

The authors have no conflict of interests related to this publication.

\section{Author contributions}

Literature search, writing of the manuscript (SMH, KKS).

\section{References}

[1] Fede G, D'Amico G, Arvaniti V, Tsochatzis E, Germani G, Georgiadis D, et al. Renal failure and cirrhosis: a systematic review of mortality and prognosis. J Hepatol 2012;56:810-818. doi: 10.1016/j.jhep.2011.10.016.

[2] Wu CC, Yeung LK, Tsai WS, Tseng CF, Chu P, Huang TY, et al. Incidence and factors predictive of acute renal failure in patients with advanced liver cirrhosis. Clin Nephrol 2006;65:28-33. doi: 10.1097/00007890-198509000-00008.

[3] Gonwa TA, McBride MA, Anderson K, Mai ML, Wadei H, Ahsan N. Continued influence of preoperative renal function on outcome of orthotopic liver transplant (OLTX) in the US: where will MELD lead us? Am J Transplant 2006;6: 2651-2659. doi: 10.1111/j.1600-6143.2006.01526.x.

[4] Formica RN, Aeder M, Boyle G, Kucheryavaya A, Stewart D, Hirose R, et al. Simultaneous liver-kidney allocation policy: a proposal to optimize appropriate utilization of scarce resources. Am J Transplant 2016;16:758-766. doi 10.1111/ajt.13631.

[5] Nair S, Verma S, Thuluvath PJ. Pretransplant renal function predicts survival in patients undergoing orthotopic liver transplantation. Hepatology 2002;35: 1179-1185. doi: 10.1053/jhep.2002.33160.

[6] Charlton MR, Wall WJ, Ojo AO, Ginès P, Textor S, Shihab FS, et al. Report of the first international liver transplantation society expert panel consensus conference on renal insufficiency in liver transplantation. Liver Transpl 2009;15: S1-S34. doi: 10.1002/It.21877.

[7] Wadei HM, Lee DD, Croome KP, Mai ML, Golan E, Brotman R, et al. Early allograft dysfunction after liver transplantation is associated with shortand long-term kidney function impairment. Am J Transplant 2016;16: 850-859. doi: 10.1111/ajt.13527.

[8] Srinivas TR, Stephany BR, Budev M, Mason DP, Starling RC, Miller C, et al. An emerging population: kidney transplant candidates who are placed on the waiting list after liver, heart, and lung transplantation. Clin J Am Soc Nephrol 2010;5:1881-1886. doi: 10.2215/CJN.02950410.

[9] Eason JD, Gonwa TA, Davis CL, Sung RS, Gerber D, Bloom RD. Proceedings of consensus conference on simultaneous liver kidney transplantation (SLK). Am J Transplant 2008;8:2243-2251. doi: 10.1111/j.1600-6143.2008.02416.x.

[10] Locke JE, Warren DS, Singer AL, Segev DL, Simpkins CE, Maley WR, et al. Declining outcomes in simultaneous liver-kidney transplantation in the MELD era: ineffective usage of renal allografts. Transplantation 2008;85:935-942. doi: 10.1097/TP.0b013e318168476d.

[11] Fong TL, Khemichian S, Shah T, Hutchinson IV, Cho YW. Combined liverkidney transplantation is preferable to liver transplant alone for cirrhotic patients with renal failure. Transplantation 2012;94:411-416. doi: 10 . 1097/TP.0b013e3182590d6b.

[12] Sharma P, Shu X, Schaubel DE, Sung RS, Magee JC. Propensity score-based survival benefit of simultaneous liver-kidney transplant over liver transplant alone for recipients with pretransplant renal dysfunction. Liver Transpl 2016; 22:71-79. doi: 10.1002/lt.24189.

[13] Choudhury RA, Reese PP, Goldberg DS, Bloom RD, Sawinski DL, Abt PL. A paired kidney analysis of multiorgan transplantation: implications for allograft survival. Transplantation 2017;101:368-376. doi: 10.1097/TP. 0000000000001151.

[14] Schnitzler MA, Whiting JF, Brennan DC, Lentine KL, Desai NM, Chapman W, et al. The life-years saved by a deceased organ donor. Am J Transplant 2005 5:2289-2296. doi: 10.1111/j.1600-6143.2005.01021.x.

[15] Organ Procurement and Transplantation Network. A Guide to calculating and interpreting the Kidney Donor Profile Index (KDPI), 2014. Available from: http://optn.transplant.hrsa.gov/ContentDocuments/Guide_to_Calculating Interpreting KDPI.pdf.

[16] Parajuli S, Foley D, Djamali A, Mandelbrot D. Renal function and transplantation in liver disease. Transplantation 2015;99:1756-1764. doi: 10 1097/TP.0000000000000820

[17] Targher G, Chonchol M, Zoppini G, Abaterusso C, Bonora E. Risk of chronic kidney disease in patients with non-alcoholic fatty liver disease: is there a link? J Hepatol 2011;54:1020-1029. doi: 10.1016/j.jhep.2010.11.007.

[18] Gonwa TA, Mai ML, Melton LB, Hays SR, Goldstein RM, Levy MF, et al. Renal replacement therapy and orthotopic liver transplantation: the role of continuous veno-venous hemodialysis. Transplantation 2001;71:1424-1428.

[19] Ojo AO, Held PJ, Port FK, Wolfe RA, Leichtman AB, Young EW, et al. Chronic renal failure after transplantation of a nonrenal organ. N Engl J Med 2003; 349:931-940. doi: 10.1056/NEJMoa021744.

[20] Ruebner R, Goldberg D, Abt PL, Bahirwani R, Levine M, Sawinski D, et al. Risk of end-stage renal disease among liver transplant recipients with pretransplant renal dysfunction. Am J Transplant 2012;12:2958-2965. doi: 10. 1111/j.1600-6143.2012.04177.x.

[21] Northup PG, Argo CK, Bakhru MR, Schmitt TM, Berg CL, Rosner MH. Pretransplant predictors of recovery of renal function after liver transplantation. Liver Transpl 2010;16:440-446. doi: 10.1002/It.22008.

[22] Israni AK, Xiong H, Liu J, Salkowski N, Trotter JF, Snyder JJ, et al. Predicting end-stage renal disease after liver transplant. Am J Transplant 2013;13: 1782-1792. doi: 10.1111/ajt.12257. 
Hussain S.M. et al: Simultaneous liver-kidney transplantation

[23] Sharma P, Goodrich NP, Schaubel DE, Guidinger MK, Merion RM. Patient-specific prediction of ESRD after liver transplantation. J Am Soc Nephrol 2013;24: 2045-2052. doi: 10.1681/ASN.2013040436

[24] Pichler RH, Huskey J, Kowalewska J, Moiz A, Perkins J, Davis CL, et al. Kidney biopsies may help predict renal function after liver transplantation. Transplantation 2016;100:2122-2128. doi: 10.1097/TP.0000000000001334.

[25] Davis CL, Feng S, Sung R, Wong F, Goodrich NP, Melton LB, et al. Simultaneous liver-kidney transplantation: evaluation to decision making. Am J Transplant 2007;7:1702-1709. doi: 10.1111/j.1600-6143.2007.01856.x.

[26] Nadim MK, Davis CL, Sung R, Kellum JA, Genyk YS. Simultaneous liverkidney transplantation: a survey of US transplant centers. Am J Transplant 2012;12:3119-3127. doi: 10.1111/j.1600-6143.2012.04176.x.

[27] Henny FC, Kleinbloesem CH, Moolenaar AJ, Paul LC, Breimer DD, van Es LA. Pharmacokinetics and nephrotoxicity of cyclosporine in renal transplant recipients. Transplantation 1985;40:261-265. doi: 10.5414/CNP65028.

[28] Naesens M, Kuypers DR, Sarwal M. Calcineurin inhibitor nephrotoxicity. Clin ] Am Soc Nephrol 2009;4:481-508. doi: 10.2215/CJN.04800908.

[29] https://optn.transplant.hrsa.gov/media/1192/0815-12_SLK_Allocation.pdf.

[30] Asch WS, Bia MJ. New organ allocation system for combined liver-kidney transplants and the availability of kidneys for transplant to patients with stage 4-5 CKD. Clin J Am Soc Nephrol 2017;12:848-852. doi: 10. 2215/CJN.08480816.

[31] Longenecker JC, Estrella MM, Segev DL, Atta MG. Patterns of kidney function before and after orthotopic liver transplant: associations with length of hospital stay, progression to end-stage renal disease, and mortality. Transplantation 2015;99:2556-2564. doi: 10.1097/TP.0000000000000767.

[32] Brennan TV, Lunsford KE, Vagefi PA, Bostrom A, Ma M, Feng S. Renal outcomes of simultaneous liver-kidney transplantation compared to liver transplant alone for candidates with renal dysfunction. Clin Transplant 2015;29: 34-43. doi: 10.1111/ctr.12479.

[33] Wadei HM, Gonwa TA, Taner CB. Simultaneous liver kidney transplant (SLK) allocation policy change proposal: is it really a smart move? Am J Transplant 2016;16:2763-2764. doi: 10.1111/ajt.13844.

[34] Pham PT, Lunsford KE, Bunnapradist S, Danovitch GM. Simultaneous liverkidney transplantation or liver transplantation alone for patients in need of liver transplantation with renal dysfunction. Curr Opin Organ Transplant 2016;21:194-200. doi: 10.1097/MOT.0000000000000299.

[35] Cheng XS, Stedman MR, Chertow GM, Kim WR, Tan JC. Utility in treating kidney failure in end-stage liver disease with simultaneous liver-kidney transplantation. Transplantation 2017;101:1111-1119. doi: 10.1097/TP. 0000000000001491.

[36] Ekser B, Mangus RS, Fridell W, Kubal CA, Nagai S, Kinsella SB, et al. A novel approach in combined liver and kidney transplantation with long-term outcomes. Ann Surg 2017;265:1000-1008. doi: 10.1097/SLA.0000000000001752.

[37] Taner T, Heimbach JK, Rosen CB, Nyberg SL, Park WD, Stegall MD. Decreased chronic cellular and antibody-mediated injury in the kidney following simultaneous liver-kidney transplantation. Kidney Int 2016;89:909-917. doi: 10. 1016/j.kint.2015.10.016. 